\title{
The impact of COVID-19 lockdowns on the genetic integrity of your mouse colonies
}

\author{
Are your mouse colonies in the best state to resume experiments? A short commentary on some key issues to \\ consider when moving forward.
}

Natalia Moncaut and Sarah Hart-Johnson

W hen the COVID-19 pandemic swept across the world last year, most mouse breeding facilities, along with everyone else, had to radically change their way of working. The rolling national lockdowns and social distancing necessitated fast thinking and pushed us to make decisions outside of our normal experience and comfort zones. Faced with the prospect of animal care shortages and concerned with whether we would have the resources to feed, clean, and provide medical care to thousands of animals, many facilities reduced their colonies in some form or another. Some Institutes and Universities stopped breeding wild type animals, relying instead on commercial breeders to supply them. Others asked their researchers to look at reducing colony size or removing tick-over colonies whilst new experiments were halted and mice could not be used within the time frame needed.

Within this context, close analysis of breeding strategies was often not possible, and the emphasis was therefore on maintaining an active line but with a minimum number of animals. However, maintaining colonies with a reduced number of individuals has the inevitable consequence of speeding up genetic drift. This is the process by which mutations accumulate in the genome over time, or the constant tendency of genes to evolve through spontaneous mutations. Whilst essential in nature, this can be a problem in science, where the need for reproducibility is key and controlling variation a principal component of experimental design.

It is estimated that spontaneous mutations accumulate at a rate of $\sim 100$ SNPs per genome every generation ${ }^{1}$. Whilst a majority of these are undetectable with no obvious phenotypic effect, a phenotypic variation can occur every two generations. After 16 generations, there is a $90 \%$ chance that two substrains differ at one or more gene loci ${ }^{2}$. Within a small, closed colony these can become fixed very quickly, compromising the reproducibility of research with those animals ${ }^{3}$. Both visible phenotypic changes and silent ones may significantly affect your experimental research outcome with impact on the biology of mice in your colony; for example, changes can occur in their immune and neuronal systems ${ }^{4,5}$. The pandemic response has resulted in a notable decrease in the number of individuals within colonies. Potentially, this could lead to a significant issue with reproducibility if this is not addressed.

So, what can you do about it? Refresh, replace, or change breeding strategies There aren't currently any reliable and affordable technologies to check for genetic drift, but if your colony has been closed or inbred for 5-10 generations, you can presume it has happened; if you're limited to one or two breeders, there is a high likelihood that those mutations have become fixed. As such, you have two options to recover or "turn back time": refreshing or replacing the colony.

Refreshing your Genetically Altered (GA) line involves crossing it back to the appropriate wild-type line obtained from a reputable source every five-to-ten generations for two or three generations. You could also keep the colony open maintaining it heterozygously if this is possible experimentally, to reduce the chance of a mutation becoming fixed.

Replacing the GA line involves cryopreserving embryos or sperm with the required genotype at a point in time, preferably right after it has been backcrossed or refreshed, and using them to entirely replace the colony every ten generations. This is more appropriate than refreshing for lines with multiple genetic modifications.

Beyond refresh and replace (though ideal, we acknowledge the time and financial consequences these approaches imply), the best way to slow genetic drift is to slow the buildup of generations within a colony. Maximising breeding lifespan and avoiding extended inbreeding within your GA colonies should slow the buildup of fixed mutations.

\section{Look to your wild type stock}

Other potential issues affecting reproducibility and the genetic integrity of your strains revolve around the use of different sources of wild type (or GA) mice. Shortages in stock during COVID-19 lockdowns might have resulted in changes to your usual supply. Have you inadvertently used a different substrain to breed with your mice, or as a control? Does the substrain you are now using have the same genetic background as your experimental GA animals? These again can lead to hugely variable results - and could be one explanation of a change in results pre- and post-COVID ${ }^{6-8}$.

Another issue gaining increasing attention is the effect of a change in microbiome $^{9-11}$. If you decided to stop in-house breeding of wild type stocks and started to import mice from different sources, or changed any other environmental conditions, you may have changed the microbiota of your mice. These changes can inadvertently introduce variables affecting all aspects of metabolism ${ }^{12-14}$. Being aware of any changes made is critical to identifying possible sources of variation.

\section{Know your mice! Have good records} To manage these risks going forward, there are a couple of key things you should be doing routinely. The most important point is to know your mouse ${ }^{8}$. Breeding pedigrees are essential to know the background of your line and when you need to refresh it, as well as accurate records on where stocks came from and how they were generated.

Where pedigrees are not available, background testing is increasingly becoming affordable, with some methods spotting genetic contamination of constructs as well as background strains. It is also essential to regularly genotype your line for the alleles of interest, at least when refreshing breeders even if they are homozygous - as this is the quickest check of any genetic contamination.

\section{Cryopreserve your lines!}

Cryopreserving mouse lines gives insurance against loss of stocks due to adverse events 
such as environmental disasters, disease outbreaks, genetic drift, transgene silencing, and breeding failure. During 2020, in case of an imminent closure of research mouse facilities around the world, archiving programs were escalated in order to have most of their mouse lines safely archived. Sperm cryopreservation in particular is a fast and effective way to secure unique alleles.

Since most of breeding programs were stopped or put in tick-over to reduce the production of unused mice, one of the most striking outcomes that came to light was the idea of using cryopreservation as a tool of colony management.

Cryopreserved embryos or sperm can be used to generate experimental age-matched cohorts. Lines intermittently needed for creating new crosses, such as deleter lines, are also good candidates for managing from the freezer if you have a good in vitro fertilization (IVF) service to hand (Fig. 1). It should be noted that culture environment and/or IVF approach may alter the expression and methylation level of imprinted genes during development and consequently this might have an impact on the generated cohort ${ }^{15,16}$.

\section{Closing Remarks}

In the immediate aftermath of the first lockdowns, as part of an NC3Rs expert working group, we contributed to a comprehensive resource hub aimed at providing advice on a range of scenarios such as interrupted experiments and reduced colony size ${ }^{17}$. Also, NC3Rs together with RSPCA will be updating the best practice guidelines on the Sharing \& Archiving of GA mice. These are a good source of information for those looking to secure their stocks against future issues ${ }^{18}$.

The COVID-19 pandemic made evident that to maintain the genetic integrity of mouse colonies, good colony management, a change in breeding strategy and archiving

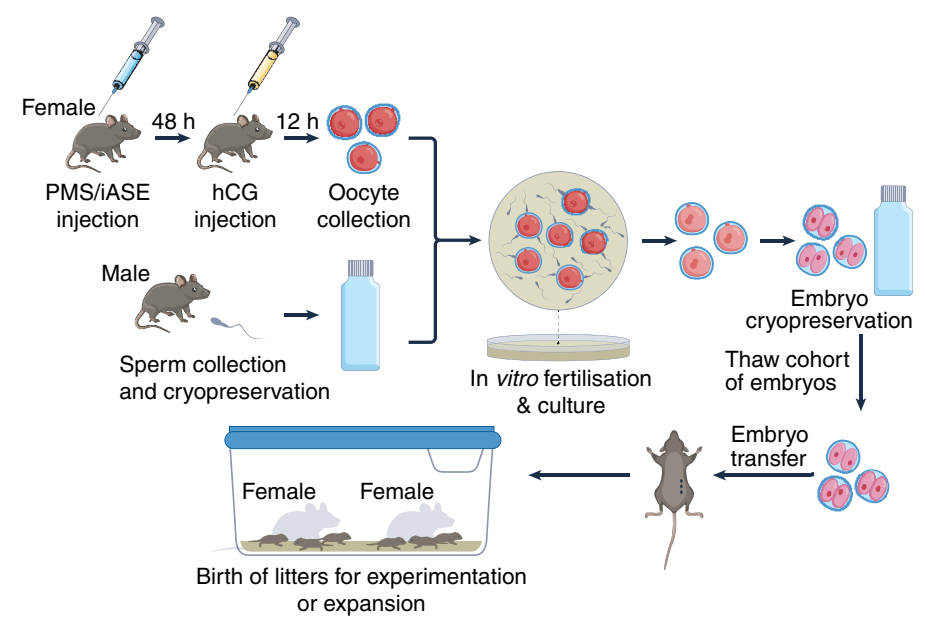

Fig. 1| Cryopreservation as a tool of colony management. Oocytes obtained after hyperovulation ${ }^{19}$ are used for IVF with frozen or fresh sperm and fertilised oocytes are cryopreserved in batches. When animals are required for experiments, embryos are thawed and transferred into a pseudopregnant female. Litters will be ready to be used as experimental cohorts.

re-evaluation was needed. Although there is no simple and universal solution to avoid the effects of genetic drift and/or changes in microbiome on the colonies phenotype and hence the science, it is important to acknowledge their existence and be aware of possible solutions to mitigate them.

Natalia Moncaut ${ }^{1 凶}$ and

Sarah Hart-Johnson ${ }^{2 凶}$

${ }^{1}$ Genome Editing and Mouse Models, Cancer Research UK Manchester Institute, University of Manchester, Manchester, UK. ${ }^{2}$ Colony Management Services, BRF, The Francis Crick Institute, London, $U K$.

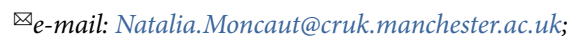
Sarah.Hart-Johnson@crick.ac.uk

Published online: 30 September 2021 https://doi.org/10.1038/s41684-021-00872-2

\section{References}

1. Lynch, M. Trends Genet. 26, 345-352 (2010).

2. Stevens, J. C. et al. Trends Mol. Med. 13, 512-519 (2007).
3. Zeldovich, L. Lab. Anim. (NY). 46, 255-257 (2017).

4. Purtha, W. E. et al. Proc. Natl Acad. Sci. USA 109, 898-904 (2012).

5. Specht, C. G. \& Schoepfer, R. BMC Neurosci. 2, 11-19, https://doi. org/10.1186/1471-2202-2-11 (2001)

6. Kelmenson, $\mathrm{P}$. There is no such thing as a C57BL/6 mouse! (2016) https://www.jax.org/news-and-insights/jax-blog/2016/june/ there-is-no-such-thing-as-a-b6-mouse

7. Mekada, K. et al. Exp. Anim. 58, 141-149 (2009).

8. Taft, R. A., Davisson, M. \& Wiles, M. V. Trends Genet. 22, 649-653 (2006).

9. Servick, K. Science https://doi.org/10.1126/science.aah7199 (2016)

10. Alegre, M.-L. Genome Biol. 20, 108-110 (2019).

11. Perry, M. N., Bello, S. M. \& Smith, S. L. Lab. Anim. (NY). 49 301-302 (2020).

12. Weger, B. D. et al. Cell Metab. 29, 362-382 (2019).

13. Kobayashi, T. et al. J. Immunol. 192, 1918-1927 (2014).

14. Kadosh, E. et al. Nature. 586, 133-138 (2020).

15. Rivera, R. M. et al. Hum Mol. Genet. 17, 1-4 (2008).

16. Aljahdali, A. et al. Hum Reprod. 35, 2497-2514 (2020).

17. NC3RS. Breeding and colony management resource hub. https:// nc3rs.org.uk/breeding-and-colony-management

18. Hurst, J. et al. Sharing and archiving of genetically altered mice: Opportunities for reduction and refinement. Report of the RSPCA Resource Sharing Working Group (RSWG), (2008). https://bbsrc ukri.org/documents/genetically-altered-mice-pdf/ [To be revised in November 2021]

19. Takeo, T. \& Nakagata, N. PLoS One 10, e0128330 (2015). 